Totrahedron Letters No. 35, pp 3369 - 3372, 1973. Porgamon Press. Printed in Great Britain.

DARSTELLUNG UND THERMOLYSE DER HEXAMETHYLDEWARBENZOL-CYCLOADDUKTE AROMATISCHER NITRILOXIDE UND DES DIPHENYLNITRILIMINS Gisela Brintrup und Manfred Christ $1^{*}$

Institut fïr Organische Chemle der Universität, 8 Minchen 2, Karlstr. 23

(Received in Germany 17 May 1973; recelved in UK for publication 17 July 1973)

Kürzlich wurde das Phenylazid-Cycloaddukt an Hexamethyldewarbenzol beschrieben (1). Das vergleichbare Ansprechen von Phenylazid und Benzonitriloxid auf die Ringspannung in Cycloalkenen (2) veranlaBte uns, aromatische Ni-

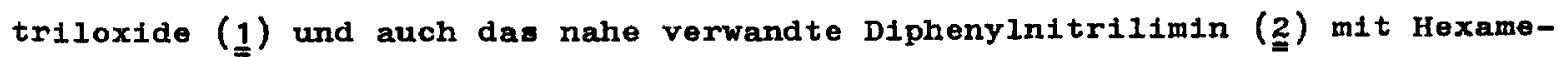
thyldewarbenzol (3) umzusetzen. Wie im Schema angegeben, bildeten sich dabel die tricyclischen 1:1-Addukte (4) bzw. (ㅌ) (3). Die Reaktionstrăgheit von 3 findet in den bescheidenen Adduktausbeuten Ihren Ausdruck.<smiles>CC1=C(C)C2(C)C3(C)C([Ge])=NOC3(C)C12C</smiles>

$\underline{4}$

\begin{tabular}{|c|c|c|c|}
\hline & Ar & $\begin{array}{c}\text { Aus b. } \\
\%\end{array}$ & $\begin{array}{l}\text { Fp. } \\
{ }^{\circ} \mathrm{C}\end{array}$ \\
\hline 40 & $\mathrm{C}_{6} \mathrm{H}_{5}$ & 17 & $103-104$ \\
\hline $4 b$ & $4-\mathrm{NO}_{2}-\mathrm{C}_{6} \mathrm{H}_{4}$ & 16 & $199-201$ \\
\hline $4 c$ & $2.4 .6-\left(\mathrm{CH}_{3}\right)_{3} \mathrm{C}_{6} \mathrm{H}_{2}$ & 11 & ÖL \\
\hline$\underline{5}$ & & 6 & $152-154$ \\
\hline
\end{tabular}

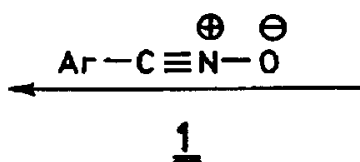

$\underline{\underline{3}}$<smiles>CC1=C(C)C2(C)C(C)=C(C)C12C</smiles>
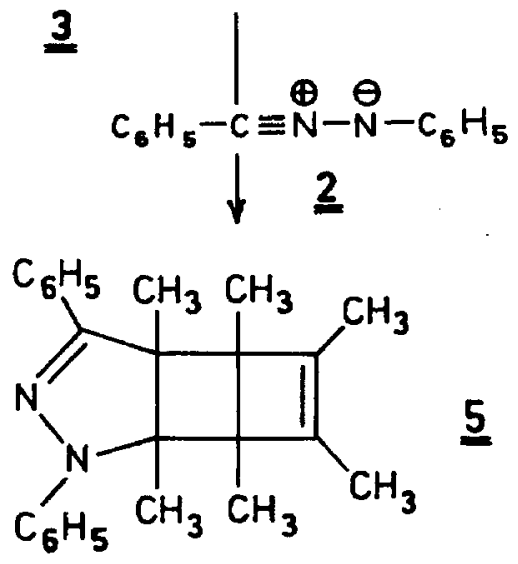

Die Charakterisierung der Verbindungen $\underline{\underline{4}}$ und $\underline{\underline{5}}$ stitzt stch auf die Massen- und die ${ }^{1}$ H-NMR-Spektren. In den Massenspektren aller Verbindungen findet man neben den Molekulpeake $\left(\mathrm{M}^{+}\right)$und denen der $\left(\mathrm{M}-\mathrm{CH}_{3}\right)$-Ionen die weitaus intensivste Linie bei $\mathrm{m} / \mathrm{e} 108$, dem Tetramethylcyclobutadienkation $\left(\mathrm{C}_{8} \mathrm{H}_{12}{ }^{+}\right)$ 3369 
entsprechend. Offenbar wird der Cyclobutanring in den Tricyclen $\mathbf{4}$ und $\mathbf{2}$ gespalten, weil so die Heteroaromaten 4.5-Dimethyl-3-ary1-isoxazol bzw. -1.3diphenyl-pyrazol entstehen, deren $(M+1)$-Ionen ebenfalls auftreten.

Im Gegensatz zu den Azid-Addukten an 2 (1) haben die Protonen der beiden olefinischen Methylgruppen im ${ }^{1}$ H-NMR-Spektrum der Verbindungen des Typs 4 nicht die gleiche chemische Verachiebung, so daB die Homoallylkopplung (4) $A_{3} B_{3}$-Multipletts verursacht. Die GröBenordnung dieser Kopplung entapricht derjenigen im Hexamethyldewarbenzol, die wir ohne Lobsungamittel anhand der ${ }^{13} \mathrm{C}$-Sateliten zu $1.3 \mathrm{~Hz}$ beatimmt haben.

Bei der Thermolyse des 3-Addukts von Phenylazid erhielten paquette und Haluska (5) bei $118^{\circ}$ als Hauptprodukt einen $10 \pi-E l$ ektronen-Neunring, nämlich das 4.5.6.7.8.9-Hexamethyl-1-pheny1-1.2.3-triazonin, ders theoretisches Interesse zukommt.

4a, $\stackrel{4 \mathrm{~b}}{\underline{\underline{a}}}$ und $\underline{\underline{5}}$ blieben nach 24 Stunden bel $130^{\circ}$ in Xy101 unverăndert. Erst 6-stüniges Erhitzen auf $200^{\circ}$ In Decalin verursachte teilweisen zerfall, der zu Hexametnylbenzol führte. Einen völlig anderen Verlauf nahm die Thermolyse vor $4 \underline{\underline{c}}$. Nach 20 stunden bel $130^{\circ}$ in Xylol hatten sich mit elner Ausbeute von $86 \%$ zwei Verbindungen im Verhältnis 20:1 gebildet, die Isomere zu 4 c sind. Durch Dickschichtchromatographie wurden boide Substanzen rein erhalten (3). Ein Kontrollverauch lehrte, daß unter den Thermolysebedingungen eine Verbindung nicht Folgeprodukt der anderen ist. Fur das Hauptprodukt [farblose Kristallo, Schmp. 58-60\%, Sdp. $140-150^{\circ}$ (Bad)/0.001 Torr] schlagen wir die bicyclische struktur 6 und fur das Nebenprodukt [farbloses öl mit Sdp. 140-150 (Bad)/0.001 Torr] die monocyclische struktur $\underline{Z}$ vor. Die folgenden spektroskopischen Daten legen dies nahe.

6: ${ }^{13} \mathrm{C}$-NMR-Spektrum (6) $\left(\mathrm{CDCl}_{3}\right.$; in ppm feldabwärts von internem TMS): 11

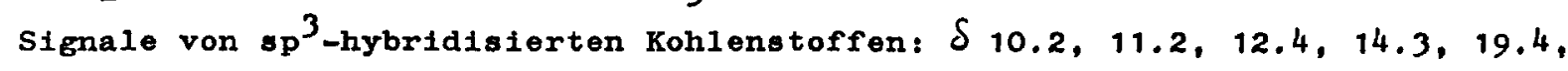
$19.7,21.1,25.0$ (Methylkohlenstoffe);41.2 (c-7);51.8 (c-3a);91.9 (c-7a). 10 Signale von sp ${ }^{2}$-hybridiaierten Kohlenstoffen: $107.8\left(\mathrm{CH}_{2}\right) ; 124.9$ (C-1'); $128.4\left(\mathrm{C}-3^{\prime}, \mathrm{C}-5^{\prime}\right) ; 137.6,137.9,138.5,139.3,140.8\left(\mathrm{C}-5, \mathrm{C}-6, \mathrm{C}-2^{\prime}, \mathrm{C}-4^{\prime}\right.$, $\left.c-6^{\prime}\right) ; 153.4(\mathrm{C}-3) ; 158.3(\mathrm{C}-4)$. Die Zahl der Absorptionen zeigt an, daB die freie Rotation des Mesitylrestes eingeschrankt sein muß, wodurch seine spie- 
gelsymmetrischen Hälten in chemisch verschiedenen Umgebungen lokalisiert werden.
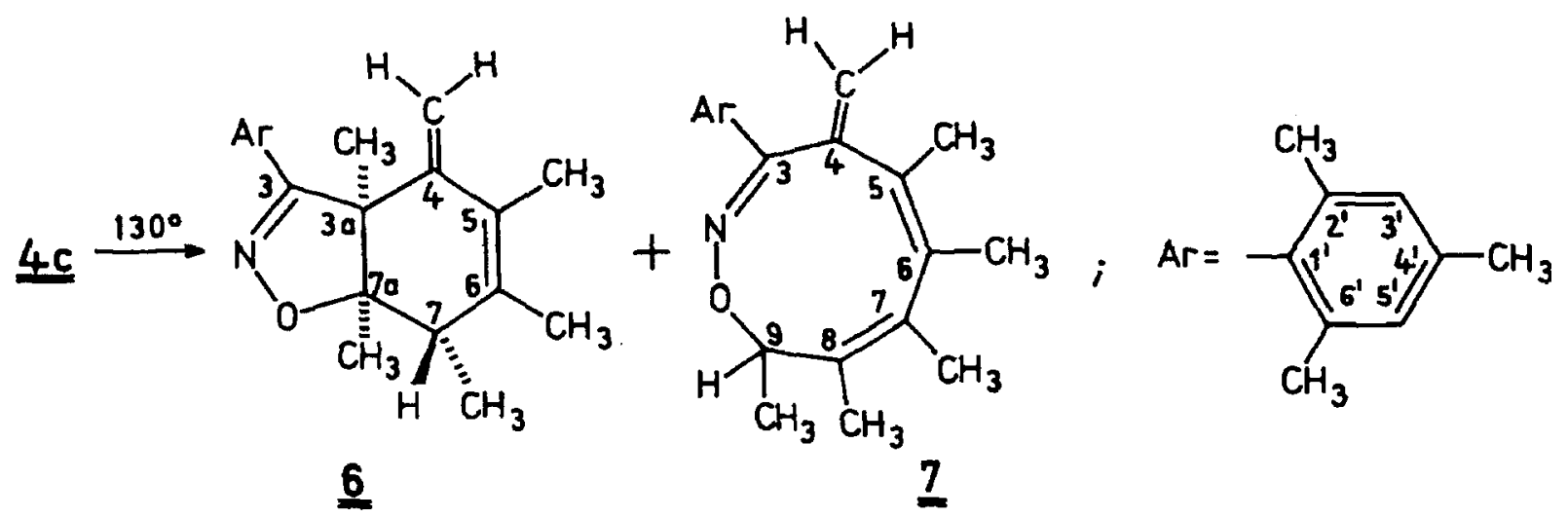

Massenspektrum: m/e $323\left(\mathrm{M}^{+}\right.$, relative Intensität $\left.33 \%\right), 308\left(\mathrm{M}^{+}-\mathrm{CH}_{3}, 29 \%\right)$, 215 (4.5-Dimethyl-3-mesityl-isoxazolkation, 37\%) 109.1036 (ber. fur $\mathrm{C}_{8} \mathrm{H}_{13}{ }^{+}$ $109.1014,100 \%)$.

${ }^{1} \mathrm{H}-\mathrm{NMR}-\mathrm{Spektrum}\left(\mathrm{CCl}_{4}\right): 3^{\prime}-\mathrm{H}, 5^{\prime}-\mathrm{H}$ s (breit) $\delta 6.84 ; 2^{\prime}-\mathrm{CH}_{3}, 6^{\prime}-\mathrm{CH}_{3}$ s.10;

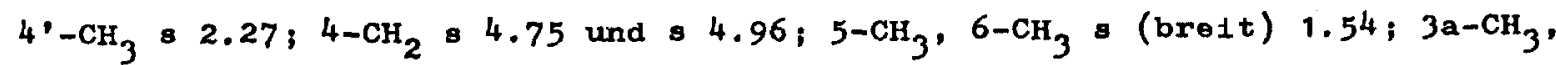
$7 \mathrm{a}-\mathrm{CH}_{3}$ \& 1.35 und $\mathrm{s} 1.07 ; 7-\mathrm{H}$ m 2.62 und $7-\mathrm{CH}_{3}$ d 0.97 mit $\mathrm{J}=7.0$ Hz. Entkoppein von $7-\mathrm{H}$ verwandelt die $7-\mathrm{CH}_{3}-\mathrm{Res}$ onanz in ein singulett. Aus der fehlenden Kopplung der Methylenprotonen zum Allylproton loiten wir die Anordnung der Substituenten im Cyclohexenring ab. Sie wird bestätigt durch einen positiven Overhauser-Effekt ( 7 ) von 10\%, den das Methylensingulett bei $\delta 4.75$ zelgt, wenn man die Resonanz der olefinischen Methylgruppen bei $1.5^{4}$ săttigt. Eine wechselsettige Signalverstärkung von $37 \%$ eines Methylenprotons bei der Entkopplung dos zweiten beweist deren geminale Anordnung. Auf die relative Orientierung der Isoxazolineinheit sowie die endo-Position des 7-H schlieBen wir aus Pseudokontaktverschiebungen, die in Gegenwart von Europium-(III) gemessen wurden.

2: ${ }^{13} \mathrm{C}-\mathrm{NMR}-\mathrm{Spektrum}(6)\left(\mathrm{CDCl}_{3}\right): 9$ Signale von sp ${ }^{3}$-Kohlenstoffen: $\delta 13.3$, $15.1,18.1,19.8,19.9,20.3,21.1,28.5$ (Methylkohlenstoffe); 90.1 (C-9). 12 Signale von sp ${ }^{2}$-Kohlenstoffen: $107.6\left(\mathrm{CH}_{2}\right) ; 128.5\left(\mathrm{C}-3^{\circ}, \mathrm{C}-5^{\prime}\right) ; 119.9$, $124.7,127.4,137.6,137.9,138.8,140.3,141.6\left(\mathrm{C}-5, \mathrm{C}-6, \mathrm{C}-7, \mathrm{C}-8, \mathrm{C}-1^{\prime}\right.$, 
$\left.\mathrm{C}-2^{\prime}, \mathrm{C}-4^{\prime}, \mathrm{C}-6^{\prime}\right) ; 154.1(\mathrm{c}-3) ; 157.9(\mathrm{c}-4)$

Massenspektrum: $\mathrm{m} / \mathrm{e} 323\left(\mathrm{M}^{+}, 25 \%\right) ; 308\left(\mathrm{M}^{+}-\mathrm{CH}_{3}, 100 \%\right) ; 280(67 \%) ; 264(100 \%)$.

${ }^{1} \mathrm{H}-\mathrm{NMR}-\mathrm{Spektrum}\left(\mathrm{CCl}_{4}\right): 3^{\prime}-\mathrm{H}, 5^{\prime}-\mathrm{H}$ (breit) \& $6.87 ; 2^{\prime}-\mathrm{CH}_{3}, 6^{\prime}-\mathrm{CH}_{3}, 2.16$; $4^{\prime}-\mathrm{CH}_{3}$ \& $2.30 ; 4-\mathrm{CH}_{2}$ \& 4.78 und 8 4.94. Die Signale aller 5 weiteren Methylgruppen erscheinen bel tieferem Feld alo jene in 6 , nämlioh alo Multiplott zwischen $\delta 1.57$ und 1.87. 9-H erscheint als verbreitertes Quadruplett mit $\mathrm{J} \approx$ $6 \mathrm{~Hz}$ bei $\& 5.14$, das bel Entkopplung aller niohtaromatischen Methylprotonen in oin scharfes Singulett ubergeht. Die Tieffeldverschiebung zeigt seine zum elektronegativen Sauerstoff benachbarte 9-Position an, wahrend die fehlende Allylkopplung zu den vinylischen Protonen die 8-stellung fir die Methylengruppe ausschlie Bt. Die Verschiebungsdifferenz von 0.16 ppm zwischen den beiden Vinylprotonenresonanzen 1st am besten mit der Methylengruppe in 4-Stellung vereinbar, da sie hier mit dem unterschledilchen Mesityl-Ringotromeinflus auf das cis- und trans-standige Proton erklärt werden kann. Die verschiedenen Thermolyseprodukte der Verbindungen $\underset{\underline{a}}{\mathrm{a}}, \underline{\mathrm{b}} \mathbf{\mathrm { b }}$ sowie 5 einerseits und $\underline{\underline{4}} \underline{\underline{c}}$ andererseits legen voneinander abweichende Reaktionsverlatufo naho. Wir vermuten, das die ortho-Methylgruppen des Mesitylrestes im Fallo von $\underline{\underline{c}} \mathrm{c}$ aufgrund ihros Raumbedarfs elne bedeutsame Rolie spielen. Die Mechanismen dieser Thermolysen sind Gegenstand weiterer Untersuchungen.

\section{LITERATUR UND ANMERKUNGEN}

(1) L.A. Paquette, R.J. Haluska, M.R. Short, L.K. Read und J. Clardy, J.Amer.chem.Soc. 24,529 (1972).

(2) K. Bast, M. Christ1, R. Huisgen und W. Mack, Chem.Ber, , 1m Druck.

(3) Alle neuen Verbindungen ergaben korrekte elementaranalysenwerte.

(4) tibersicht: M. Barfield und S. Sternhell, J.Amer.chem.Soc. 24, 1905 (1972)

(5) L.A. Paquette und R.J. Haluska, J.Amer.chem.Soc. 24.534 (1972).

(6) Den Herren Priv.-Doz. Dr. J. Firl, Technische Universitat Mlinchen, und Dr. B.L. Hawkins, California Institute of Technology, Pasadena, danken wir fur die Aufnahme der ${ }^{13} \mathrm{C}-\mathrm{NMR}-$ Spektren.

(7) Utbersicht: J.H. Noggle und R.E. Schirmer, The Nuclear Overheuser Effect: Chemical Applications, Academic Press, New York 1971. 